\title{
ON THE NUMBER OF LIMIT CYCLES IN PERTURBATIONS OF A QUADRATIC REVERSIBLE CENTER
}

\author{
JUANJUAN WU ${ }^{凶}$, LINPING PENG and CUIPING LI \\ (Received 1 January 2011; accepted 27 February 2012; first published online 11 October 2012) \\ Communicated by J. J. Koliha

\begin{abstract}
This paper is concerned with the bifurcation of limit cycles from a quadratic reversible system under polynomial perturbations. It is proved that the cyclicity of the period annulus is two, and also a linear estimate of the number of zeros of the Abelian integral for the system under polynomial perturbations of arbitrary degree $n$ is given.
\end{abstract}

2010 Mathematics subject classification: primary 34C07; secondary 34C08, 37G15.

Keywords and phrases: Abelian integral, limit cycles, cyclicity, quadratic reversible system.

\section{Introduction}

The weak version of Hilbert' 16th problem, asking what is the number of limit cycles for the polynomial perturbation of planar systems with centers, is still open even for the quadratic case (see [1]). Zoladek [14] classified planar quadratic systems with at least one center into four types, $Q^{H}, Q_{3}^{R}, Q_{3}^{L V}$ and $Q_{4}$, and conjectured that the cyclicity of the periodic annulus of quadratic systems is two. The conjecture has been proved completely for the cases $Q^{H}$ and $Q_{4}$ (see [2, 5, 6]). Because of the rich dynamical behaviors, mathematicians focus on the case $Q_{3}^{R}$. For $Q_{3}^{R} \backslash Q^{H}$, since the integrating factor is no longer a constant, the problem is much more difficult. After an affine transformation, any system of $Q_{3}^{R} \backslash Q^{H}$ can be written as

$$
\left\{\begin{array}{l}
\dot{x}=a x^{2}+b y^{2}-2(b-1) y+b-2, \\
\dot{y}=-2 x y
\end{array}\right.
$$

where $a, b \in R$. Excellent results on limit cycles of system (1.1) under quadratic perturbations have been obtained in recent years. In [3], the authors considered the case $a=-1 / 2$ and $\{b:-1<b<1\}$, and they presented the upper bound of the limit

(c) 2012 Australian Mathematical Publishing Association Inc. 1446-7887/2012 \$16.00 
cycles and the corresponding bifurcation diagrams. The case where $a=-3 / 2$ has been investigated in $[9,13]$ : by using some complex methods, C. Liu (unpublished) proved that four limit cycles could be found for $\{b: 0<b<2\}$ with $(3,1)$-configuration. Liang [9] and Zhao [13] considered the cases where $\{b: b<0, b=2\}$ and $\{b: b>2\}$, and proved that the least upper bound of the number of limit cycles is two.

In this paper, we will study the case where $a=-3 / 4$ and $b=0$ in system (1.1), which after an affine transformation becomes

$$
\left\{\begin{array}{l}
\dot{x}=x y \\
\dot{y}=\frac{3}{8} y^{2}+\frac{9}{2^{10}}(1-x) .
\end{array}\right.
$$

Our main results are the following theorems.

THEOREM 1.1. Under quadratic perturbations, the exact upper bound on the number of limit cycles bifurcating from the period annulus of integrable system (1.2) is two, that is, the cyclicity of the periodic annulus of system (1.2) is two.

THEOREM 1.2. Under polynomial perturbations of arbitrary degree $n$, the upper bound of the number (taking multiplicities into account) of limit cycles arising from the period annulus of integrable system (1.2) does not exceed $30 n-37$.

As a complement to [3, 9, 13], Theorem 1.1 partly verifies the conjecture given in [14] for system (1.2), while Theorem 1.2 improves the linear estimate $30 n+20$ of the zeros of the associated Abelian integrals in [11].

\section{The Abelian integral and Picard-Fuchs equation}

As a planar integrable system, there is a $C^{1}$ function which is constant along any solution curve of the system, and the integrable system (1.2) has first integral

$$
H(x, y)=x^{-3 / 4}\left(\frac{1}{2} y^{2}+\frac{9}{2^{8}} x+\frac{3}{2^{8}}\right)=h
$$

with the integrating factor $M(x, y)=x^{-7 / 4}$.

We denote the closed connected component of the real curve $H(x, y)=h$ by $\Gamma_{h}$, defined on the maximal open interval $S=\left(3 / 2^{6},+\infty\right)$. The phase portrait of system (1.2) in the Poincaré disk is shown in Figure 1.

Lemma 2.1. Under quadratic perturbations, the Abelian integral $I(h)$ related to system (1.2) can be expressed as

$$
I(h)=\alpha I_{0}(h)+\beta I_{1}(h)+\gamma I_{-1}(h),
$$

where $\alpha, \beta$ and $\gamma$ are constants, and $I_{i}(h)=\oint_{\Gamma_{h}} x^{i-7 / 4} y d x$ with $i=-1,0$ and 1 .

Proof. The conclusion follows directly from [8, Theorem 2]. 


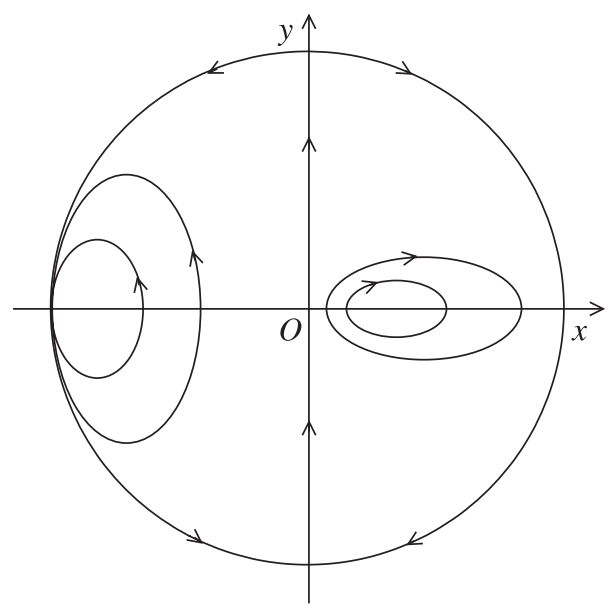

Figure 1. Phase portrait of system (1.2) in the Poincaré disk.

By the standard method in [2], we get the Picard-Fuchs equation in the following lemma.

Lemma 2.2. For system (1.2), the vector function $J(h)=\operatorname{col}\left(I_{0}(h), I_{1}(h), I_{-1}(h)\right.$, $\left.I_{1 / 4}(h)\right)$ satisfies the Picard-Fuchs equation as follows:

$$
J(h)=M_{1}(h) J^{\prime}(h),
$$

where

$$
M_{1}(h)=\left(\begin{array}{cccc}
h & 0 & 0 & -\frac{3}{2^{6}} \\
0 & \frac{1}{3} h & 0 & -\frac{1}{2^{6}} \\
-\frac{6}{7} h & 0 & \frac{3}{7} h & \frac{9}{7 \times 2^{6}} \\
\frac{3^{5}}{5 \times 2^{20} h^{2}} & -\frac{3^{5}}{2^{20} h^{2}} & \frac{2 \times 3^{4}}{5 \times 2^{20} h^{2}} & \frac{3}{2} h
\end{array}\right) .
$$

Lemma 2.3. The ratio $\varphi(h) / h$ satisfies the equation

$$
\left(\frac{\varphi(h)}{h}\right)^{\prime}=\left(m+\frac{2^{8}}{3} k-\frac{6 k \mathcal{H}}{h^{2}}\right) I_{0}^{\prime \prime}(h)+\left(n-\frac{2 k \mathcal{H}}{3 h^{2}}\right) I_{-1}^{\prime \prime}(h)
$$

where $\varphi(h)=I(h)-h I^{\prime}(h) / 3, \mathcal{H}=3^{5} \times 2^{-20} h^{-2} / 5, m, n$ and $k$ are constants relevant to $\alpha, \beta$ and $\gamma$.

Proof. Differentiating (2.2) with respect to $h$ yields

$$
M_{2}(h) J^{\prime}(h)=M_{1}(h) J^{\prime \prime}(h),
$$


where

$$
M_{2}(h)=\left(\begin{array}{cccr}
0 & 0 & 0 & 0 \\
0 & \frac{2}{3} & 0 & 0 \\
\frac{6}{7} & 0 & \frac{4}{7} & 0 \\
\frac{3^{5}}{5 \times 2^{19} h^{3}} & -\frac{3^{5}}{2^{19} h^{3}} & \frac{2 \times 3^{4}}{5 \times 2^{19} h^{3}} & -\frac{1}{2}
\end{array}\right)
$$

The first and second rows of (2.4) give

$$
\begin{gathered}
I_{1 / 4}^{\prime \prime}(h)=\frac{2^{6}}{3} h I_{0}^{\prime \prime}(h), \\
I_{1}^{\prime}(h)=\frac{1}{2} h I_{1}^{\prime \prime}(h)-\frac{3}{2^{7}} I_{1 / 4}^{\prime \prime}(h) .
\end{gathered}
$$

Substituting (2.5) and (2.6) into the third row of (2.4),

$$
\frac{3}{2} h I_{-1}^{\prime \prime}(h)-\frac{3}{2} h I_{0}^{\prime \prime}(h)=3 I_{0}^{\prime}(h)+2 I_{-1}^{\prime}(h) .
$$

Similarly, the first row of (2.4) combined with (2.5)-(2.7) yields

$$
I_{1 / 4}^{\prime}(h)=6 \mathcal{H} I_{0}^{\prime \prime}(h)+\frac{2}{3} \mathcal{H} I_{-1}^{\prime \prime}(h)-2{ }^{6} h^{2} I_{0}^{\prime \prime}(h)
$$

By using the Picard-Fuchs function, we get

$$
\begin{aligned}
I(h)= & \left(\alpha-\frac{6}{7} \gamma\right) h I_{0}^{\prime}(h)+\frac{1}{3} \beta h I_{1}^{\prime}(h)+\frac{3}{7} \gamma h I_{-1}^{\prime}(h) \\
& +\left(-\frac{3}{2^{6}} \alpha-\frac{1}{2^{6}} \beta+\frac{9}{2^{6} \times 7} \gamma\right) I_{1 / 4}^{\prime}(h) .
\end{aligned}
$$

Meanwhile, differentiating both sides of (1.2) with respect to $h$ directly yields

$$
I^{\prime}(h)=\alpha I_{0}^{\prime}(h)+\beta I_{1}^{\prime}(h)+\gamma I_{-1}^{\prime}(h) .
$$

From (2.9) and (2.10), we get

$$
\varphi(h)=\left(\frac{2}{3} \alpha-\frac{6}{7} \gamma\right) h I_{0}^{\prime}(h)+\frac{2}{21} \gamma h I_{-1}^{\prime}(h)+\left(-\frac{3}{2^{6}} \alpha-\frac{1}{2^{6}} \beta+\frac{9}{2^{6} \times 7} \gamma\right) I_{1 / 4}^{\prime}(h),
$$

which implies that

$$
\left(\frac{\varphi(h)}{h}\right)^{\prime}=m I_{0}^{\prime \prime}(h)+n I_{-1}^{\prime \prime}(h)+\frac{k}{h} I_{1 / 4}^{\prime \prime}(h)-\frac{k}{h^{2}} I_{1 / 4}^{\prime}(h)
$$

where

$$
m=2 \alpha / 3-6 \gamma / 7, \quad n=2 \gamma / 21, \quad k=-3 \times 2^{-6} \alpha-2^{-6} \beta+9 \times 2^{-6} \gamma / 7 \text {. }
$$

Substituting (2.5) and (2.8) into (2.11), we get (2.3). 
REMARK 2.4. From equation (2.4), we obtain

$$
G(h) J^{\prime \prime}(h)=M_{3}(h) J^{\prime}(h),
$$

where $G(h)=h\left(16777216 h^{4}-81\right)$ and

$$
M_{3}(h)=\left(\begin{array}{cccc}
\frac{81}{5} & 0 & \frac{54}{5} & -262144 h^{3} \\
\frac{81}{5} & 33554432 h^{4}-16 & \frac{54}{5} & -262144 h^{3} \\
33554432 h^{4}-\frac{729}{5} & 0 & \frac{67108864}{3} h^{4}-\frac{486}{5} & -262144 h^{3} \\
\frac{1728}{5} h & 0 & \frac{1152}{5} h & -\frac{16777216}{3} h^{4} .
\end{array}\right) .
$$

The first array of (2.12) yields

$$
G(h) I_{0}^{\prime \prime}(h)=\frac{1}{5} f(h),
$$

where

$$
f(h)=81 I_{0}^{\prime}(h)+54 I_{-1}^{\prime}(h)-1310720 h^{3} I_{1 / 4}^{\prime}(h) .
$$

By direct calculation, we get

$$
G(h) f^{\prime}(h)=-f^{2}(h)+\frac{G(h)}{h} f(h)+g(h) .
$$

where

$$
g(h)=1310720 h^{2}\left(67108864 h^{5} / 3-108 h-3\right) I_{1 / 4}^{\prime}(h) / I_{0}^{\prime}(h) .
$$

Obviously $f\left(3 / 2^{6}\right)=0$, then $f^{\prime}\left(3 / 2^{6}\right)=\left.(g(h) / G(h))\right|_{h=3 / 2^{6}}$. If $f(h)$ has another zero point for $h \in S$, without loss of generality, we suppose that $h \in S$ is adjacent to $3 / 2^{6}$ such that $f\left(h_{0}\right)=0$, then $f^{\prime}\left(h_{0}\right) f^{\prime}\left(3 / 2^{6}\right)<0$, which contradicts $g(h)>0$ for $h \in\left[3 / 2^{6},+\infty\right)$.

Hence $f(h)$ has no zero point for $h \in S$, which implies that $I_{0}^{\prime \prime}(h) \neq 0$.

Lemma 2.5. The ratio $v(h)=I_{-1}^{\prime \prime}(h) / I_{0}^{\prime \prime}(h)$ satisfies the Riccati equation as follows:

$$
\tilde{G}(h) v^{\prime}(h)=-a_{12} v^{2}(h)+\left(a_{22}-a_{11}\right) v(h)+a_{21},
$$

where $\tilde{G}(h)=6 h\left(16777216 h^{4}-81\right)$ and

$$
\left(a_{i j}\right)_{2 \times 2}=\left(\begin{array}{cc}
-729-234881024 h^{4} & -81 \\
-2187+67108864 h^{4} & -243+33554432 h^{4}
\end{array}\right) .
$$

Proof. Differentiating (2.7) and (2.8) with respect to $h$, we get

$$
\begin{gathered}
-I_{0}^{\prime \prime \prime}(h)+I_{-1}^{\prime \prime \prime}(h)=3 h^{-1} I_{0}^{\prime \prime}(h)+\frac{1}{3} h^{-1} I_{-1}^{\prime \prime}(h), \\
\left(6 \mathcal{H}-2^{6} h^{2}\right) I_{0}^{\prime \prime \prime}(h)+\frac{2}{3} \mathcal{H} I_{-1}^{\prime \prime \prime}(h)=\left(\frac{7}{3} \times 2^{6} h-6 \mathcal{H}^{\prime}(h)\right) I_{0}^{\prime \prime}(h)+\frac{2}{3} \mathcal{H}^{\prime}(h) I_{-1}^{\prime \prime}(h) .
\end{gathered}
$$

The definition of $v(h)$ implies that

$$
v^{\prime}(h)=\frac{I_{-1}^{\prime \prime \prime}(h)}{I_{0}^{\prime \prime}(h)}-v(h) \frac{I_{0}^{\prime \prime \prime}(h)}{I_{0}^{\prime \prime}(h)} .
$$

Substituting (2.14a) and (2.14b) into (2.15), we get Lemma 2.5. 


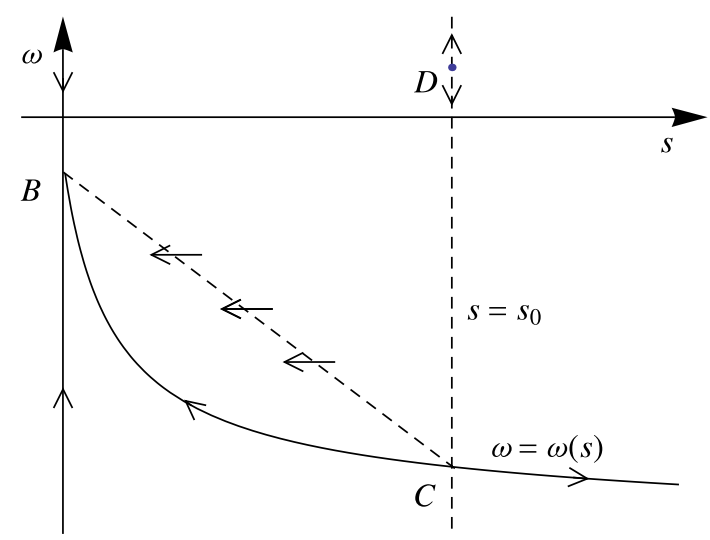

FIGURE 2. Behaviors of system (3.1).

\section{Global behaviors of the auxiliary curve and centroid curve}

Let $s=h^{4}, \omega(s)=v(h)$, the Riccati function (2.13) is equivalent to the twodimensional system

$$
\left\{\begin{array}{l}
\dot{s}=24 s(16777216 s-81) \\
\dot{\omega}=81 \omega^{2}+(486+268435456 s) \omega+67108864 s-2187
\end{array}\right.
$$

which has four singularities: two saddles $A(0,3)$ and $C(81 / 16777216,-23)$, a stable node $B(0,-9)$ and an unstable node $D(81 / 16777216,1)$; see Figure 2 . We denote the auxiliary curve on the $(s, \omega)$-plane by $C_{\omega}$ :

$$
C_{\omega}=\{(s, \omega(s)): \omega(s)=v(h), s \in(81 / 16777216,+\infty)\} .
$$

Lemma 3.1. $C_{\omega}$ is located on the unstable manifold of the saddle $C$.

Proof. Let $P(h)=I_{1}(h) / I_{0}(h), Q(h)=I_{-1}(h) / I_{0}(h), R(h)=I_{1 / 4}(h) / I_{0}(h)$. Since the level curve $\Gamma_{h}$ shrinks to the center $(1,0)$ as $h$ tends to $3 / 2^{6}$, by the mean value theorem, $P(h), Q(h)$ and $R(h)$ converge to 1 as $h$ tends to $3 / 2^{6}$.

Differentiating (2.2) with respect to $h$, we obtain

$$
\tilde{G}(h) J^{\prime \prime}(h)=M_{4}(h) J(h),
$$

where

$$
M_{4}(h)=\left(\begin{array}{cccc}
\frac{1701}{5 h} & -\frac{729}{h} & \frac{1134}{5 h} & -1048576 h^{2} \\
-\frac{1701}{5 h} & \frac{9\left(81+67108864 h^{4}\right)}{h} & -\frac{1134}{5 h} & 5242880 h^{2} \\
\frac{7\left(-2187+335544320 h^{4}\right)}{5 h} & \frac{6561}{h} & \frac{6561}{h} & 9437184 h^{2} \\
\frac{36288}{5} & -15552 & \frac{24192}{5} & -\frac{67108864}{3} h^{3}
\end{array}\right) .
$$


We denote $M_{4}(h)$ by the form $\left(b_{i j}\right)_{4 \times 4}$. From the first and third arrays of equation (3.2), we get

$$
v(h)=\frac{b_{31}+b_{32} P(h)+b_{33} Q(h)+b_{34} R(h)}{b_{11}+b_{12} P(h)+b_{13} Q(h)+b_{14} R(h)} .
$$

Substituting (2.1) into (3.3), we obtain $\omega(81 / 16777216)=-23$.

Therefore, we get the stable and unstable manifolds of the saddle $C$ :

$$
\begin{gathered}
W_{C}^{s}=\left\{(s, \omega(s)): s=\frac{81}{16777216}\right\}, \\
W_{C}^{u}=\left\{(s, \omega(s)): \omega(s)=v(h), s \in\left(\frac{81}{16777216},+\infty\right)\right\} .
\end{gathered}
$$

This concludes the proof.

Moreover, we notice that there exists an isoclinic line $\omega=\tilde{\omega}(s)$ defined by $\dot{\omega}=0$ between $B$ and $C$. Comparing the slope of $C_{\omega}$ with $\omega=\tilde{\omega}(s)$, we can extend $C_{\omega}$ from $C$ to $B$ and determine its position (see Figure 2).

Lemma 3.2. $C_{\omega}$ is globally convex and strictly decreasing.

Proof. Since the curve $C_{\omega}$ is located on the unstable manifold of the saddle $C$, we can suppose that $\omega(s)$ has the following expansion near $C(81 / 16777216,-23)$ :

$$
\omega(s)=-23+\omega_{1}\left(s-\frac{81}{16777216}\right)+\frac{\omega_{2}}{2 !}\left(s-\frac{81}{16777216}\right)^{2}+\cdots .
$$

According to system (3.1), we substitute (3.4) into the equation

$$
\dot{\omega}-\tilde{G}(h) \frac{d \omega}{d s}=0
$$

From (3.5), we obtain

$$
\omega_{1}=-\frac{381681664}{234}, \quad \omega_{2}=\frac{74841557479456768}{531441} .
$$

Hence, the curve $\mathcal{C}_{\omega}$ is convex near the left endpoint.

Next, we claim that $C_{\omega}$ is globally convex. Otherwise, without loss of generality, we suppose that $C_{\omega}$ has an inflection points right to $s=81 / 16777216$, then we find a straight line $L$ on the $(s, \omega)$-plane such that it cuts $C_{\omega}$ at at least three points and intersects $s=81 / 16777216$ below the saddle point $(81 / 16777216,-23)$, leading to at least three points on $L$, at which the vector field (3.1) is tangent to $L$. This contradicts the fact that $\left.(\dot{\omega}-a \dot{s})\right|_{\omega=a s+b}$ is quadratic in $s$, where $a, b$ are arbitrary constants. Therefore the curve $C_{\omega}$ is globally convex for $s \in(81 / 16777216,+\infty)$.

Using a horizontal line $\omega=\omega_{0}$ instead of the straight line $L$, a similar argument verifies the monotonicity of $C_{\omega}$.

We denote the centeriod curve on the $(P, Q)$-plane by $\Sigma=\{(P, Q)(h): h \in S\}$.

Lemma 3.3. The curve $\Sigma$ is increasing. 
Proof. Using arguments as in [10, Theorem 2], we obtain $P^{\prime}(h)>0, Q^{\prime}(h)>0$ for $h \in S$, which implies Lemma 3.3.

Lemma 3.4. The curve $\Sigma$ is concave for $0<h-3 / 2^{6} \ll 1$.

Proof. From (2.2), we obtain

$$
\tilde{G}(h) J^{\prime}(h)=M_{5}(h) J(h),
$$

where

$$
M_{5}(h)=\left(\begin{array}{cccc}
-\frac{4131}{5}+100663296 h^{4} & 2187 & -\frac{1134}{5} & 3145728 h^{3} \\
-\frac{1701}{5} & 9\left(81+33554432 h^{2}\right) & -\frac{1134}{5} & 3145728 h^{3} \\
-\frac{6561}{5}+201326592 h^{4} & 2187 & -\frac{6804}{5}+234881024 h^{4} & 3145728 h^{3} \\
-\frac{36288}{5} h & 46656 h & -\frac{24192}{5} h & 67108864 h^{4}
\end{array}\right) .
$$

For convenience, we denote $M_{5}(h)$ as $\left(c_{i j}\right)_{4 \times 4}$. From (3.6), we get the fourdimensional system

$$
\left\{\begin{array}{l}
\dot{h}=\tilde{G}(h) \\
\dot{P}=c_{21}+c_{22} P+c_{23} Q+c_{24} R-P\left(c_{11}+c_{12} P+c_{13} Q+c_{14} R\right), \\
\dot{Q}=c_{31}+c_{32} P+c_{33} Q+c_{34} R-Q\left(c_{11}+c_{12} P+c_{13} Q+c_{14} R\right), \\
\dot{R}=c_{41}+c_{42} P+c_{43} Q+c_{44} R-R\left(c_{11}+c_{12} P+c_{13} Q+c_{14} R\right) .
\end{array}\right.
$$

The asymptotic expansions of $P(h)$ and $Q(h)$ can be expressed near the hyperbolic singular point $\left(3 / 2^{6}, 1,1,1\right)$ as

$$
\begin{aligned}
& P(h)=1+p_{1}\left(h-\frac{3}{2^{6}}\right)+p_{2}\left(h-\frac{3}{2^{6}}\right)^{2}+\cdots, \\
& Q(h)=1+q_{1}\left(h-\frac{3}{2^{6}}\right)+q_{2}\left(h-\frac{3}{2^{6}}\right)^{2}+\cdots .
\end{aligned}
$$

For simplicity, we take the calculation of the coefficient of $P(h)$ as an example. Notice that $P^{\prime}(h) \dot{h}-\dot{P}=0$. Substituting (3.7) and (3.8) into it, we get

$$
p_{1}=\frac{64}{3}, \quad p_{2}=\frac{78848}{243}
$$

Similarly,

$$
q_{1}=\frac{320}{9}, \quad q_{2}=\frac{148480}{729} .
$$

Hence

$$
\left.\frac{d^{2} \tilde{Q}}{d P^{2}}\right|_{h=3 / 2^{6}}=-\frac{20}{27}
$$

Therefore, Lemma 3.4 holds.

Lemma 3.5. The curve $\Sigma$ is concave for $0<1 / h \ll 1$. 
Proof. Since $\Gamma_{h}$ is symmetric with respect to the $x$-axis, we need only consider the part above the $x$-axis. Denoting the intersection points of $\Gamma_{h}$ with the $x$-axis by $\left(\xi_{h}, 0\right)$ and $\left(\eta_{h}, 0\right)$, where $0<\xi_{h}<1<\eta_{h}$, we can estimate $\xi_{h}$ and $\eta_{h}$ as $h$ tends to positive infinity,

$$
\xi_{h} \sim\left(3^{-1} \times 2^{8} \times h\right)^{-4 / 3}, \quad \eta_{h} \sim\left(3^{-2} \times 2^{8} \times h\right)^{4} .
$$

From the definition of $I_{0}(h)$,

$$
\begin{aligned}
I_{0}(h)= & 2 \int_{\xi_{h}}^{\eta_{h}} x^{-7 / 4} \sqrt{2 h x^{3 / 4}-\frac{9}{2^{7}} x-\frac{3}{2^{7}}} d x \\
= & 2^{3 / 2} h^{1 / 2} \int_{\xi_{h}}^{\eta_{h}} x^{-11 / 8} d x \\
& \quad+\frac{1}{2} \int_{\xi_{h}}^{\eta_{h}} x^{-11 / 8} \sum_{n=1}^{+\infty} \frac{\frac{1}{2}\left(\frac{1}{2}-1\right) \cdots\left(\frac{1}{2}-n+1\right)}{n !}\left(-\frac{9}{2^{8}} x^{1 / 4}-\frac{3}{2^{8}} x^{-3 / 4}\right)^{n} h^{-n} d x \\
= & a_{0} h+a_{1} h^{-1 / 3}+o\left(h^{-1}\right)
\end{aligned}
$$

where $a_{0}=2^{15 / 2} 3^{-3 / 2} B(1 / 2,3 / 2)>0, a_{1}=-2^{25 / 6} 3^{5 / 6} B(1 / 6,1 / 2)<0$ and $B(\alpha, \beta)$ is the beta function.

By a similar calculation,

$$
\begin{gathered}
I_{1}(h)=b_{0} h^{3}+b_{1} h^{-1 / 3}+o\left(h^{-3}\right), \\
I_{-1}(h)=c_{0} h^{7 / 3}+c_{1} h^{-1 / 3}+o\left(h^{-5 / 3}\right),
\end{gathered}
$$

where $b_{0}=2^{47 / 2} 3^{-5} B(5 / 2,3 / 2)$ and $c_{0}=2^{109 / 6} 3^{-17 / 6} B(11 / 6,3 / 2)$.

The expansion series of $I_{i}(h)$ in $h$ with $i=0,1$ and -1 show that, as $h$ tends to positive infinity,

which implies that

$$
P(h) \sim \frac{b_{0}}{a_{0}} h^{2}, \quad Q(h) \sim \frac{c_{0}}{a_{0}} h^{4 / 3},
$$

$$
\frac{d^{2} Q}{d p^{2}} \sim-\frac{2 a_{0} c_{0}}{9 b_{0}^{2}} h^{-8 / 3}
$$

Thus the statements of Lemma 3.5 holds.

\section{Proof of Theorem 1.1}

We denote the maximum number (taking multiplicities into account) of zeros of function $I(h)$ for $h \in S$ by \#\{I(h),S\}. Since $h \neq 0$ and $I\left(3 / 2^{6}\right)=0$,

$$
\begin{aligned}
\#\{I(h), S\} & =\#\left\{h^{-3} I(h), S\right\} \\
& \leq \#\left\{-\frac{3}{h^{4}} \varphi(h), S\right\} \\
& \leq \#\left\{\left(\frac{\varphi(h)}{h}\right)^{\prime}, S\right\}+1 .
\end{aligned}
$$


(a)

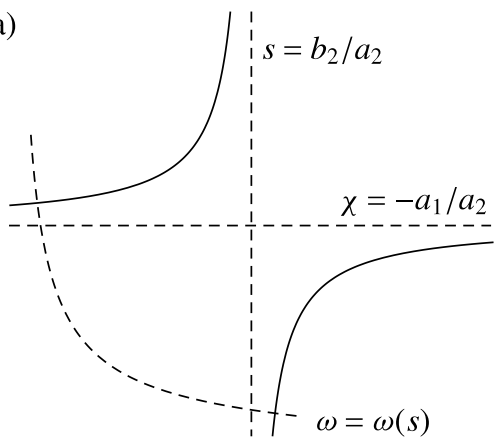

(b)

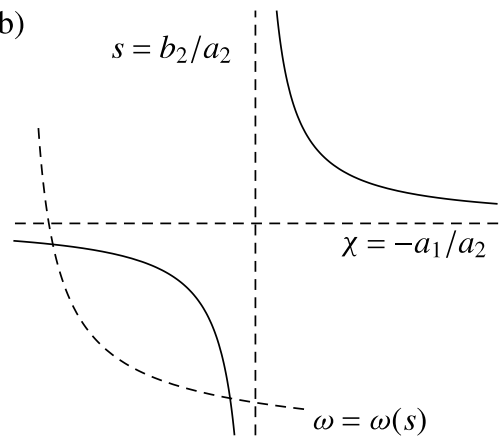

FIGURE 3. Behaviors of $C_{\omega}$ and $C_{\chi}$.

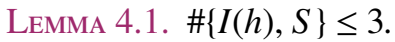

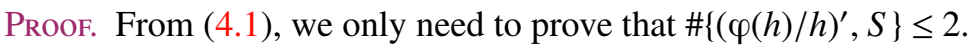

Equation (2.9) can be rewritten as follows:

$$
\left(\frac{\varphi(h)}{h}\right)^{\prime}=\frac{I_{0}^{\prime \prime}(h)}{5 \times 2^{20} h^{4}}\left(\left(5 \times 2^{20}\left(m+\frac{2^{8}}{3} k\right) h^{4}-6 k\right)+\left(5 \times 2^{20} n h^{4}-\frac{2}{3} k\right) \frac{I_{-1}^{\prime \prime}(h)}{I_{0}^{\prime \prime}(h)}\right) .
$$

Let

$$
\Phi(s)=\left(a_{1} s-b_{1}\right)+\left(a_{2} s-b_{2}\right) \omega(s),
$$

where $a_{1}=5 \times 2^{20}\left(m+2^{8} k / 3\right), b_{1}=6 k, a_{2}=5 \times 2^{20} n$, and $b_{2}=2 k / 3$. Obviously, the following equality holds:

$$
\#\left\{\left(\Phi(s),\left(\frac{81}{16777216},+\infty\right)\right\}=\#\left\{\left(\frac{\varphi(h)}{h}\right)^{\prime}, S\right\} .\right.
$$

If $a_{2} s-b_{2}=0$, then $\Phi(s)$ has at most one zero point.

When $a_{2} s-b_{2} \neq 0$, from (4.2) we can obtain

$$
\Phi(s)=\left(a_{2} s-b_{2}\right)(-\chi(s)+\omega(s))
$$

where $\chi(s)=-\left(a_{1} s-b_{1}\right) /\left(a_{2} s-b_{2}\right)$. Hence, \#\{( $\left.\Phi(s),(81 / 16777216,+\infty)\right\}$ is equal to the number of intersection points of $C_{\chi}$ and $C_{\omega}$, where $C_{\chi}$ is the graphic of $\chi=\chi(s)$ for $s \in(81 / 16777216,+\infty)$.

For $a_{1} b_{2}-a_{2} b_{1}=0$, it is easy to see that $C_{\chi}$ is a straight line and cuts $C_{\omega}$ at at most at one point. For $a_{1} b_{2}-a_{2} b_{1} \neq 0$, without loss of generality, suppose that $a_{2} \neq 0$; then $C_{\chi}$ is a hyperbola with two asymptotes $s=b_{2} / a_{2}$ and $\chi(s)=-a_{1} / a_{2}$. If $C_{\chi}$ is increasing, by the monotonicity of $C_{\omega}$, we conclude they have at most two intersection points (see Figure 3(a)). If $C_{\chi}$ is decreasing, shown in Figure 3(b), by the monotonicity of $C_{\omega}$, it only intersects with one branch of the hyperbola. If it happens to the left branch, because the convexities of the two curves $C_{\omega}$ and $C_{\chi}$ are different, they have at most 


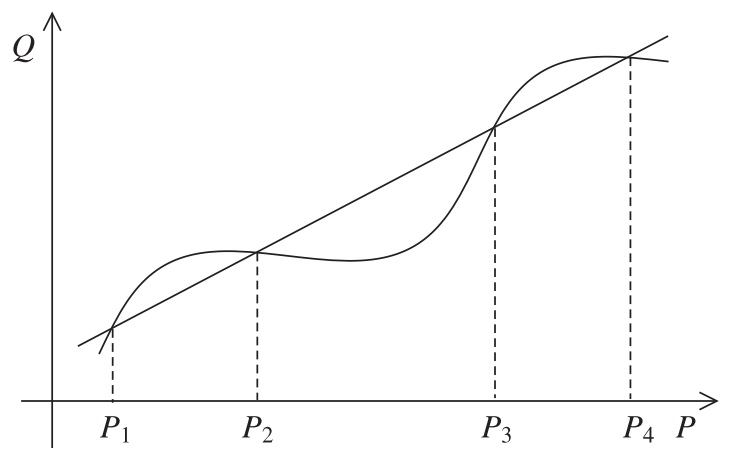

FiguRE 4. Behaviors of $\Sigma$.

two intersection points. If it happens to the right branch, since $\omega(s)$ satisfies the twodimensional system (3.1), between any two intersection points of $C_{\chi}$ with $C_{\omega}$, there is at least one point on $C_{\chi}$ at which the tangent direction of this curve coincides with the vector field (3.1). This corresponds to a zero of the function

$$
\left.\left(\frac{\dot{\omega}}{\dot{s}}-\chi^{\prime}(s)\right)\right|_{\omega=\chi(s)}=\frac{\alpha_{1} s^{2}+\beta_{1} s+\gamma_{1}}{24(16777216 s-81)\left(a_{2} s-b_{2}\right)^{2}},
$$

where $\alpha_{1}, \beta_{1}, \gamma_{1}$ are constants relevant to $a_{1}, a_{2}, b_{1}, b_{2}$. Equation (4.3) shows that there are at most two such points on $C_{\chi}$, which implies that $C_{\chi}$ cuts $C_{\omega}$ at most three times. Noticing that $\omega(0)=\chi(0)=-9$, we can conclude that they have at most two intersection points for $s \in(81 / 16777216,+\infty)$.

In summary, $C_{\chi}$ has at most two intersection points with $C_{\omega}$. This completes the proof of Lemma 4.1 .

\section{Lemma 4.2. The centeriod curve $\Sigma$ is globally concave without zero curvature.}

Proof. Because of the monotonicity of $\Sigma$, we assert that the curve $\Sigma$ is globally located on the right of the tangent line of $\Sigma$ at the left endpoint. Otherwise, there must exist another point on $\Sigma$ near the left endpoint, such that the tangent line of $\Sigma$ at this point cuts two more points of $\Sigma$, that is, $I(h)$ has at least four zeros for $h>3 / 2^{6}$, which contradicts Lemma 4.1. On the other hand, since $\Sigma$ is increasing and has the same concavity at two endpoints, if it has inflection points, they must happen in pairs. Without loss of generality, we assume that there are two inflection points in $h \in S$; then we can find an increasing straight line $L_{\alpha_{0}, \beta_{0}, \gamma_{0}}$ such that it cuts $\Sigma$ at at least three points. Suppose that their abscissas are $P_{1}, P_{2}$ and $P_{3}$ (see Figure 4 ). Then $L_{\alpha_{0}, \beta_{0}, \gamma_{0}}$ is below $\Sigma$ when $0<P(h)-P_{3} \ll 1$. Because the slope of $\Sigma$ converges to zero as $h$ tends to positive infinity, there will be another intersection point of $\Sigma$ with the increasing straight line $L_{\alpha_{0}, \beta_{0}, \gamma_{0}}$, that is, $I(h)$ has at least four zeros, which also leads to the same contradiction to Lemma 4.1. Hence $\Sigma$ has no inflection points. Moreover, we can assert 
that $\Sigma$ has no quadruple tangency points, otherwise, we obtain the same contradiction as above. In this way, we complete the proof of Lemma 4.2.

Proof of Theorem 1.1. Because of convexity and monotonicity of the centroid curve $\Sigma$, any straight line $L_{\alpha, \beta, \gamma}=\{(P, Q): \alpha+\beta P+\gamma Q=0\}$ has at most two intersection points with $\Sigma$, that is, $I(h)$ has at most two zeros. Moreover, there exist $\alpha^{*}, \beta^{*}$ and $\gamma^{*}$, such that $L_{\alpha^{*}, \beta^{*}, \gamma^{*}}$ cuts $\Sigma$ at exactly two points. We conclude that two is the exact upper bound for the number of limit cycles bifurcating from system (1.2) under small quadratic polynomial perturbations.

\section{Proof of Theorem 1.2}

After making the transformation $x=\bar{x}^{4}, y=\bar{y}$ (which has a unique value for $x \geqslant 0$ ) and changing back to $(x, y)$, the system (1.2) and its first integral (2.1) become

$$
\begin{gathered}
\left\{\begin{array}{l}
\dot{x}=\frac{1}{4} x y, \\
\dot{y}=\frac{3}{8} y^{2}+\frac{9}{2^{10}}\left(1-x^{4}\right),
\end{array}\right. \\
H(x, y)=x^{-3}\left(\frac{1}{2} y^{2}+3 p x^{4}+p\right)=h,
\end{gathered}
$$

with the integrating factor $M(x, y)=x^{-4}, p=3 / 2^{8}$. We denote the family of closed curves surrounding the center $(1,0)$ by $\Pi_{h}=\{(x, y): H(x, y)=h\}$, where $h \in S$.

Lemma 5.1. Under arbitrary perturbations of degree $n$, the Abelian integral $V(h)$ related to system (5.1) can be expressed as

$$
I(h)=\alpha(h) J_{-1}(h)+\beta(h) J_{0}(h)+\gamma(h) J_{1}(h)+\delta(h) J_{2}(h),
$$

where $\alpha(h), \beta(h), \gamma(h)$ and $\delta(h)$ are real polynomials in $h$, and $J_{i}(h)=\oint_{\Pi_{h}} x^{i-4} y d x$ with $i=-1,0,1$ and 2 . If $n \geq 4$ is even,

$\operatorname{deg} \alpha(h)=2 n-4, \quad \operatorname{deg} \beta(h)=2 n-5, \quad \operatorname{deg} \gamma(h)=2 n-6, \quad \operatorname{deg} \delta(h)=2 n-3 ;$

if $n \geq 4$ is odd,

$\operatorname{deg} \alpha(h)=2 n-5, \quad \operatorname{deg} \beta(h)=2 n-6, \quad \operatorname{deg} \gamma(h)=2 n-7, \quad \operatorname{deg} \delta(h)=2 n-4$.

Moreover, if $n=3$,

$$
\operatorname{deg} \alpha(h)=1, \quad \operatorname{deg} \beta(h)=\operatorname{deg} \gamma(h)=0, \quad \operatorname{deg} \delta(h)=2 .
$$

The proof of Lemma 5.1 is similar to that of Proposition 2.1 in [12], so we omit it. By similar arguments to Lemmas 2.1 and 2.2, we obtain the following lemmas.

Lemma 5.2. For system (5.1), $V(h)=\operatorname{col}\left(J_{-1}(h), J_{0}(h), J_{1}(h), J_{2}(h)\right)$ satisfies the Picard-Fuchs equation

$$
V(h)=M(h) V^{\prime}(h),
$$


where

$$
M(h)=\left(\begin{array}{cccc}
\frac{3}{4} h & -3 p & 0 & 0 \\
0 & h & -4 p & 0 \\
0 & 0 & \frac{3}{2} h & -6 p \\
-4 p & 0 & 0 & h
\end{array}\right) .
$$

Lemma 5.3. The ratio $v(h)=J_{-1}^{\prime \prime}(h) / J_{0}^{\prime \prime}(h)$ satisfies the Riccati equation

$$
G(h) v(h)^{\prime}(h)=-\frac{16}{3} p^{2} h v^{2}(h)+\frac{2}{3} h^{3} v(h)(h)-\frac{16}{3} h p^{2},
$$

where $G(h)=h^{4}-256 p^{4}$.

Lemma 5.4. The function $I(h)$ has the representation

$$
\delta_{2}(h) I(h)=\delta_{1}(h) J^{\prime}(h)+\Delta(h),
$$

where

$$
\Delta(h)=\alpha_{3}(h) J_{-1}^{\prime}(h)+\beta_{3}(h) J_{0}^{\prime}(h)+\gamma_{3}(h) J_{1}^{\prime}(h) .
$$

Proof. From (5.2) and (5.3), we get

$$
I(h)=\alpha_{1}(h) J_{-1}^{\prime}(h)+\beta_{1}(h) J_{0}^{\prime}(h)+\gamma_{1}(h) J_{1}^{\prime}(h)+\delta_{1}(h) J_{2}^{\prime}(h) .
$$

Differentiating (5.6) with respect to $h$ directly yields

$$
I^{\prime}(h)=\alpha_{2}(h) J_{-1}^{\prime}(h)+\beta_{2}(h) J_{0}^{\prime}(h)+\gamma_{2}(h) J_{1}^{\prime}(h)+\delta_{2}(h) J_{2}^{\prime}(h) .
$$

Eliminating $J_{2}^{\prime}(h)$ from (5.6) and (5.7), we get (5.5).

Lemma 5.5. Denote $\Delta_{1}(h)=\left(\Delta(h) / \beta_{3}(h)\right)^{\prime}$, the function $v(h)$ satisfies

$$
\frac{\Delta_{1}(h)}{J_{1}^{\prime}(h)}=\frac{\alpha_{4}(h) v(h)+v_{4}(h)}{G(h) \beta_{3}^{2}(h)} .
$$

Proof. Differentiating (5.1) with respect to $h$ yields

$$
G(h)\left(\begin{array}{l}
J_{-1}^{\prime \prime}(h) \\
J_{0}^{\prime \prime}(h) \\
J_{1}^{\prime \prime}(h) \\
J_{2}^{\prime \prime}(h)
\end{array}\right)=\left(\begin{array}{cc}
\frac{1}{3} h^{3} & -\frac{16}{3} h p^{2} \\
\frac{64}{3} p^{3} & -\frac{4}{3} h^{2} p \\
\frac{16}{3} h p^{2} & -\frac{1}{3} h^{3} \\
\frac{4}{3} h^{2} p & -\frac{64}{3} p^{3}
\end{array}\right)\left(\begin{array}{c}
J_{-1}^{\prime}(h) \\
J_{1}^{\prime}(h)
\end{array}\right) .
$$

From the definition of $\Delta(h)$ and (5.9), we get (5.8).

Proof of Theorem 1.2. Letting $\Delta_{2}(h)=\alpha_{4}(h) v(h)+\gamma_{4}(h)$, and substituting it into (5.4),

$$
G(h) \alpha_{4}(h) \Delta_{2}^{\prime}(h)=-\frac{16}{3} p h^{2} \Delta_{2}^{2}(h)+R_{1}(h) \Delta_{2}+R_{2}(h),
$$

where

$$
\begin{gathered}
R_{1}(h)=\frac{32}{3} p^{2} h^{2} \gamma_{4}(h)+G(h) \alpha_{4}^{\prime}(h)+\frac{2}{3} h^{3} \alpha_{4}(h), \\
R_{2}(h)=-\frac{16}{3} p^{2} h\left(\gamma_{5}^{2}(h)+\alpha_{4}^{2}(h)\right)-\frac{2}{3} h^{3} \alpha_{4}(h) \gamma_{4}(h)+G(h)\left(\gamma_{4}^{\prime}(h) \alpha_{4}(h)-\gamma_{4}(h) \alpha_{4}^{\prime}(h)\right) .
\end{gathered}
$$


By the methods of [7, Lemma 4.2], from (5.5) and (5.10) we obtain

$$
\begin{gathered}
\# I(h) \leqslant \# \Delta(h)+\# \delta_{2}(h)+1, \\
\# \Delta_{2}(h) \leqslant \# G(h)+\# \alpha_{4}(h)+\# R_{2}(h)+1 .
\end{gathered}
$$

The definitions of $\Delta(h)$ and $\Delta_{2}(h)$ yield

$$
\# \Delta(h) \leqslant \# \Delta_{1}(h)+1=\# \Delta_{2}(h)+1 .
$$

Then we get

$$
\# I(h) \leqslant \# G(h)+\# \alpha_{4}(h)+\# R_{2}(h)+\# \delta_{2}(h)+2 .
$$

Theorem 1.2 follows from the degrees of $\alpha_{4}(h), R_{2}(h)$ and $\delta_{2}(h)$.

\section{Acknowledgement}

The authors wish to thank the referees for their valuable comments which led to improvements to this paper.

\section{References}

[1] V. I. Arnold, 'Ten problems', Adv. Soviet Math. 1 (1990), 1-8.

[2] F. Chen, C. Li, J. Llibre and Z. Zhang, 'A unified proof on the weak Hilbert 16th problem for $n=2$ ', J. Differential Equations 221 (2006), 309-342.

[3] B. Coll, C. Li and R. Prohens, 'Quadratic perturbations of a class of quadratic reversible systems with two centers', Discrete Contin. Dyn. Syst. 24 (2009), 1-31.

[4] S. Gautier, L. Gavrilov and I. D. Iliev, 'Perturbations of quadratic centers of genus one', arXiv:0705.1609, 2007.

[5] L. Gavrilov, 'The infinitesimal 16th Hilbert problem in the quadratic case', Invent. Math. 143 (2001), 449-497.

[6] E. Horozov and I. D. Iliev, 'On the number of limit cycles in perturbations of quadratic Hamiltonian systems', Proc. Lond. Math. Soc. 69 (1994), 198-224.

[7] E. Horozov and I. D. Iliev, 'Linear estimate for the number of zeros of Abelian integrals with cubic Hamiltonians', Nonlinearity 11 (1998), 1521-1537.

[8] I. D. Iliev, 'Perturbations of quadratic centers', Bull. Sci. Math. 122 (1998), 107-161.

[9] H. Liang and Y. Zhao, 'Quadratic perturbations of a class of quadratic reversible systems with one center', Discrete Contin. Dyn. Syst. 27 (2010), 325-335.

[10] C. Li and Z. Zhang, 'A criterion for determining the monotonicity of the ratio of two Abelian integrals', J. Differential Equations 124 (1996), 407-424.

[11] W. Li, Y. Zhao, C. Li and Z. Zhang, 'Abelian integrals for quadratic centers having almost all their orbits formed by quartics', Nonlinearity 15 (2002), 863-885.

[12] Y. Zhao, W. Li, C. Li and Z. Zhang, 'Linear estimate of the number of zeros for Abelian integrals for quadratic centers having almost all their orbits formed by cubics', Sci. China (Series A) $\mathbf{4 5}$ (1998), 964-974.

[13] Y. Zhao and H. Zhu, 'Bifurcation of limit cycles from a non-Hamiltonian quadratic integrable system with homoclinic loop', Fields Inst. Commun. to appear.

[14] H. Zoladek, 'Quadratic systems with center and their perturbations', J. Differential Equations 109 (1994), 223-278. 
JUANJUAN WU, School of Math and System Sciences, Beihang University, Beijing, 100191, PR China

e-mail: magic709@ss.buaa.edu.cn

LINPING PENG, School of Math and System Sciences, Beihang University, Beijing, 100191, PR China

e-mail: penglp@buaa.edu.cn

CUIPING LI, School of Math and System Sciences, Beihang University, Beijing, 100191, PR China

e-mail: cuipingli@buaa.edu.cn 